\title{
Faecal Consistency: A Measure of Gastrointestinal Parasitic Infections in Goats
}

Dinesh Kumar Sharma, Kumaresan Gururaj, Gopal Dass, Nitika Sharma, Anil Kumar Mishra, Rajveer Singh Pawaiya, Ashok Kumar, Souvik Paul ${ }^{1}$

\begin{abstract}
Background: Gastrointestinal $(\mathrm{GI})$ parasitism in goats is a serious and widely distributed problem. Diarrhoea, moderate or severe, though a result of various factors involving feed, fodder, seasons and state of gut physiology of digestion, is a common symptom in goats infested with GI parasites, the nematodes and coccidia. Intensity of diarrhoea corresponds to quantum and type of parasitism. Methods: In the present study, 1451 faecal samples collected from goats of different ages ( $<3 \mathrm{M},>3-6 \mathrm{M},>6-12 \mathrm{M}$ and $>12 \mathrm{M}$ ) were categorized as normal, lumpy and diarrheic based on their consistency and were processed for parasitic infections. The GI parasitic load was quantified by faecal eggs/oocysts counting (FEC and FOC) per gram of faeces through the modified Mac-master technique. Identification of parasites was based on the morphology of third-stage larvae procured by coproculture of randomly selected positive samples. FEC/FOC data (strongyle and coccidian), being skewed and uneven, were log-transformed to normalize. The transformed data $\left[\log _{\mathrm{e}}(100+n)\right]$ was analyzed statistically and results were interpreted.

Result: Means of FEC and FOC in three type of faecal samples viz. normal, lumpy and diarrheic, were significantly different. Similarly, the effect of age on FEC and FOC was significant. The interaction between animals age $\times$ faecal sample type was significant in coccidian and non-significant in strongyle infection. The results were discussed to support the hypothesis that faecal consistency was a measure of coccidian and strongyle worm load in goats and can be a basis of targeted selective treatment.
\end{abstract}

Key words: Faecal consistency, Gastrointestinal parasites, Goats.

\section{INTRODUCTION}

Goats are important ruminant livestock species widely distributed over the globe. The qualities like prolificacy, hardiness, adaptability and maneuverability make goats quite popular animals to adopt. Being a sustainable producing animal, the goats are considered all-time money, meat and milk (ATM) by poor farmers during economic hardship, especially in the Afro-Asian region. But, gastrointestinal (GI) parasites are one of the serious concern in the production of goats as they, being nibbler by nature, are quite prone to $\mathrm{GI}$ parasitic infections as compared to grazer sheep (Smith and Sherman, 2009).

Among GI parasites, infections of GI nematodes and coccidia are quite common (Gonzalez- Garduno et al. 2018; Kaur et al. 2019) and at times lead to serious production losses in the form of morbidity and mortality (if not addressed properly) in goats particularly in developing countries. Clinical syndromes of both these infections are characterized majorly by anaemia and diarrhoea along with production losses.

The most popular approach for GI parasites management is to assess the parasitic load and determine the need for treatment of infected animals. The quantification of parasite propagules, like eggs/oocysts, per gram of faeces (e.g. EPG (OPG), is most commonly used technique to estimate the load (Le Jambre et al. 2007) and the susceptibility of GI parasitism (Gonzalez-Garduno et al. 2018). However, faecal EPG/OPG, being a laboratory-based method, is neither practical and nor on-spot test. So, more convenient and userfriendly approaches like FAMACHA chart and faecal
ICAR-Central Institute for Research on Goats, Makhdoom, Farah281 122, Mathura, Uttar Pradesh, India.

${ }^{1}$ ICAR-National Research Centre on Pig, Guwahati-781 015, Assam, India.

Corresponding Author: Dinesh Kumar Sharma, ICAR-Central Institute for Research on Goats, Makhdoom, Farah-281 122, Mathura, Uttar Pradesh, India. Email: Ikofwb@yahoo.co.in

How to cite this article: Sharma, D.K., Gururaj, K., Dass, G., Sharma, N., Mishra, A.K., Pawaiya, R.S., Kumar, A. and Paul, S. (2022). Faecal Consistency: A Measure of Gastrointestinal Parasitic Infections in Goats. Indian Journal of Animal Research. DOI: 10.18805/IJAR.B-4417.

Submitted: 03-02-2021 Accepted: 02-01-2022 Online: 10-01-2022

consistency score (FCS), based on morbidity markers, have been developed and used for GI parasites management (Gordon, 1967; Kaplan et al. 2004; Reynecke et al. 2009). Both these, FAMACHA chart and FCS approaches indirectly assess the $\mathrm{Gl}$ parasites burden based on most common and observable clinical symptoms of GI parasitism i.e. anaemia and diarrhoea. However, both these approaches have been used more for sheep than goats and FAMACHA chart is specifically for haemonchosis management.

The FAMACHA chart has been applied in several farm studies (Burke et al. 2007; Hassum 2014; Torres- Acosta et al. 2014; Cintra et al. 2018), yet the FCS has a meager mention in literature and need to be investigated at farm level to reduce the parasitism and cost of its management. 
Diarrhoea, though not always, is a common clinically observed symptom in goats in GI parasitic infections. The consistency of diarrheic faeces may be varied depending on the type of infection, intensity of infection and the extent of damage in gastrointestinal tract due to parasites. In field conditions, farmers treat diarrheal goats, in the first instance, with anthelmintics without diagnosing, though laboratory diagnosis is a prerequisite for therapy. Such practice of treatment goes in vain and becomes fruitlessly extravagant when diarrhoea is originated from other pathogens or the parasitic load is such that it does not need treatment. Further, excessive and indiscriminate use of anthelmintics is likely to result in anthelmintics resistance among parasites that need to be avoided and discouraged.

The present study was envisaged to revisit the epidemiology of GI parasitic infections in goats and establish a relationship, if any, between faecal consistency and faecal eggs/ oocysts counts (FEC/ FOC) per gram of faeces (EPG/ OPG). The information so generated would, however, be a useful and easy tool to be used by farmers to decide whether treatment is needed or not. It would further facilitate targeted selective and judicious use of anthelmintic and anticoccidials in goats to slow down the pace of drug resistance development.

\section{MATERIALS AND METHODS}

\section{Location of study}

The study was conducted during 2019-2020 at ICAR-Central Institute for Research on Goats (ICAR-CIRG) Makhdoom, Mathura, India located at $27^{\circ}$ North latitude and $78^{\circ}$ East latitude (MSL-176 m) in ravines of river Yamuna. The climate prevailed in the area is classified as tropical (semi-arid zone). The topography of the grazing land was undulated and area scattered over 350 acres with sandy soil and vegetation composed of natural pasture and bushes mainly dominated by natural and cultivated Zyziphus zizuba, Cenchrus ciliaris, Terminalia sp., Tephrosia purpurea, Sida rhambipholea, Achyranthes aspera, Dichrostachy bipinnata, Sacharum munja, Cyperus rotundus, Cynodon dactylon and Boehrvia diffusa. Meteorological data of this location during the study period showed temperature variation from 14 to $44^{\circ} \mathrm{C}$ in summer and 5 to $30^{\circ} \mathrm{C}$ in winter.

\section{Animals}

The animals used were goats of different age and sex from different farms in the Institute. These animals were maintained in a semi-intensive system of goat management with a defined schedule. Kids were weaned at 90 days of age. For the rest of life, animals were allowed 5-6 hrs. of grazing during the day time. While in the stall, the productive, pregnant does and breeding male (in breeding season) were provided $200-400 \mathrm{~g}$ of concentrate feed depending on their size and physiological stage. The animals were dewormed pre and post-monsoon selectively as per annual health schedule. The anthelmintic groups were changed every year to restrict their excessive use.

\section{Faeco-physical and parasitological examination}

The faecal samples were procured regularly (during 201819) for monitoring the worm load and to ascertain the need for treatment. The faecal samples were collected aseptically per rectum in polythene bags. The collection time was scheduled at 3.30 PM when animals returned from grazing and availability of staff was ensured. The faecal samples so received after collection from goat farms were graded, based on appearance, in three categories as normal, lumpy and diarrheic depending on their consistency state (Fig 1). A total of 10 samples from each faecal sample types were processed for moisture content by drying them in the crucible through hot air oven using a standard protocol (APHA, 2005) and the range of water content was framed for three faecal sample types i.e. normal, lumpy and diarrheic. The samples were processed for parasitological examination immediately after arrival in the laboratory or stored at $4{ }^{\circ} \mathrm{C}$ in the refrigerator for further processing but not later than $24 \mathrm{hrs}$. of the collection. The parasitological procedure of faecal eggs/oocysts (FEC/FOC) counting was as per Modified Mcmaster egg counting technique (MOFF, 1977).

\section{Statistical analysis}

The FEC/FOC data so generated, as observed skewed and not evenly distributed, was log-transformed to normalize it. For normalizing the data was processed by adding 100 to total FEC/FOC and the sum was transformed by natural loge transformation [ $\left.\log _{e}(E P G / O P G+100)\right]$. All the statistical analysis was applied to the transformed data. The FEC/OPG results were back-transformed to the exponential (antilogarithms) of the least-squares means, subtracting 100 and then presenting them in the results as geometric mean. The log-transformed data were analyzed using the mixed model least-squares analysis for fitting constants (Harvey, 1990). The following model was used:

$$
Y i j k=\mu+S i+A j+(S \times A) i j+e i j k
$$

Where,

Yijk is the record for the $k^{\text {th }}$ kid, $\mu$ is the overall mean, $S i$ is the fixed effect of the $i^{\text {th }}$ sample types, $A j$ is the fixed effect of the $j^{\text {th }}$ age of kids, $(S \times A) i j$ is the interaction effect between $i^{\text {th }}$ sample types and $j^{\text {th }}$ age of kids and eijk is the residual error element with standard assumptions.

To compare the different sub-groups means, Duncan's multiple range test (DMRT) as described by Kramer (1957) was applied.

\section{RESULTS AND DISCUSSION}

A total of 1451 samples, collected from a similar number of goats were processed for different coccidian (FOC) and gastrointestinal nematode infections (FEC). Of the normal, lumpy and diarrheic faecal sample types, the moisture content fell in the range of $40-70,70-85,>85$ percent respectively (Fig 1). Coccidian infection, as identified by morphological and sporulation characteristics, was of mixed nature involving several Eimeria species viz. Eimeria arloingi, E. christenseni, 

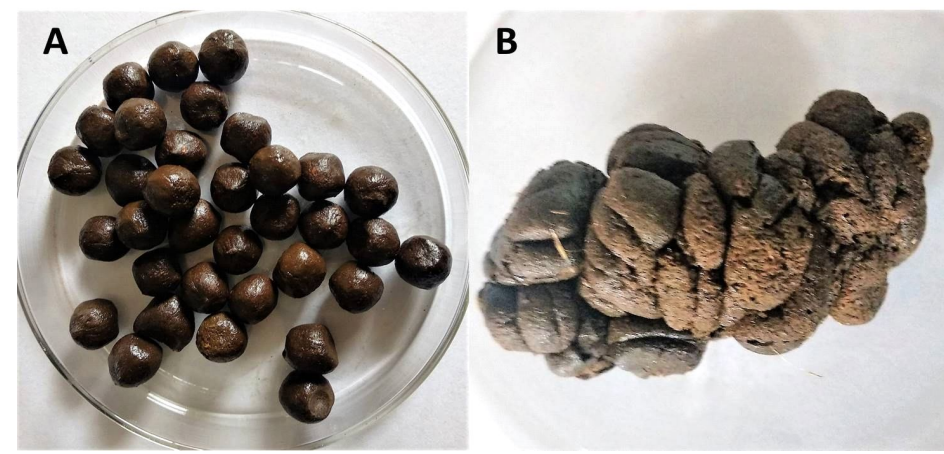

\section{c}

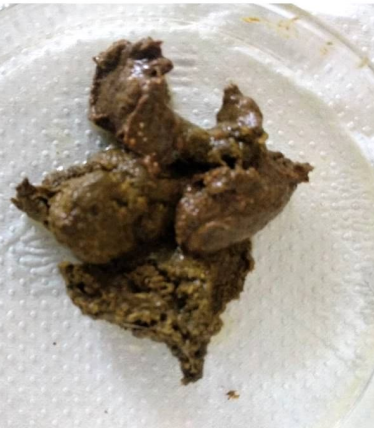

Fig 1: Faecal consistency scores (FCS) A. Normal, with intact separate pellets. B. Lumpy, with pellets start losing their shape and intactness of identity C. Diarrheic, with shape and identity of pellets is lost totally and faeces starts looking pasty and fluidic.

E. ninakohlyakaemovae, E. mitis, E. caprina, E. hirci etc. Based on coproculture examinations, Haemonchus sp. infection was predominant (97.20\%) among gastrointestinal nematodes which included the sporadic occurrence of Oesophagostomum (1.40\%), Strongyloides (0.80\%) and Trichuris $(0.60 \%)$ species also. The results of analyzed FEC data have been presented in the Table 1.

Analysis of data showed that three consistency based faecal sample types were significantly different in OPG/ EPG $(P<0.01)$ (Table 1). While FOC in diarrheic sample type $(4.831 \pm 0.021)$ was highest, the samples type with normal consistency had least FOC $(4.707 \pm 0.013)$. Similarly, the FEC per gram of faeces in the diarrheic sample type $(4.625 \pm 0.003)$ was highest while the least FEC was in normal consistency type (Table 1 ).

Age-wise analysis of data on coccidian infection showed that age was an important factor influencing OPG. The faecal OPG count was significantly higher in goats less than one year of age compared to adults. Of the younger age groups, animals up to $3 \mathrm{M}$ age showed significantly higher FOC than the $>3-6 \mathrm{M}$ and $>6-12 \mathrm{M}$ age groups. However, both $>3-6 \mathrm{M}$ and $>6-12 \mathrm{M}$ age groups were statistically similar in faecal oocysts output. The analysis of data showed that, in coccidian infection, the interaction of faecal sample types $X$ age of the animals was also significant $(P<0.01)$.

The effect of animal age was significant on per gram FEC in natural strongyle infections in goats (Table 1). Among all four age groups, mean EPG in adults was highest though it was statistically similar to EPG observed in the $>6-12 \mathrm{M}$ age group. The EPG values in lower age groups (up to $3 \mathrm{M}$ and $>3-6 \mathrm{M}$ ) were significantly lower. The FEC of consistency based sample types were also significantly different $(P<0.01)$ and the diarrheic samples had significantly higher mean FEC than the other two categories of samples. The interaction between consistency based faecal sample type and animal age, in respect of EPG, was statistically non-significant.

Consistency of faeces reflects the state of the digestive system and its physiology. Any discrepancy/anomaly of the process of digestion caused by infection (of any nature) or change in nutrition is likely to affect the consistency of faeces. Further presence of gastrointestinal parasitism is also likely to induce the peristaltic movement in the gut resulting indigestion as well as a change in faecal consistency. Moisture range in the present study was comparable and as described by others. The moisture content of normal faeces ranged between 60-65 percent in sheep and goats (Schlink et al. 2010). Bentounsi et al. (2012) reported 60, 75 and $85 \%$ moisture content in normal, soft and diarrheic faeces of sheep, respectively. The higher moisture content of faeces, than normal, can be attributed to faster passage rate or due to reduced water absorption capacity of large intestine resulted by various factors including intestinal worms (Waghorn et al. 1999). While McEwan et al. (1992) showed a positive correlation between loose faeces and dag score, Wesselink et al. (1995) described that nematodes contribute scouring in sheep. Similarly, Broughan and Wall (2007) found a strong relationship between diarrhoea and FEC. They also described that lower dry matter (i.e. higher moisture content) was significantly associated with increased faecal soiling or dag and can be an alternative way to quantify diarrhoea.

The significant effect of faecal consistency on FOC in the present study was in line and supported by Bayew (2019) who described that higher prevalence of the coccidiosis in calves with diarrheic faecal consistency than normal faecal consistency and attributed diarrheal consistency to major damage in the intestinal wall. Also, Gibbons et al. (2016) reported higher faecal oocysts count in goats with diarrheic faeces. Contrarily, Gaddam (2005) and Saratsis et al. (2011) reported no significant or weak negative relationship between faecal consistency and faecal oocysts counts respectively, in calves and lambs.

Higher EPG in diarrheic faecal sample type, like in the present study, was also reported by some other workers (Broughan and Wall 2007; Abebe et al. 2010; Bentounsi et al. 2012). Jacobson et al. (2009) also reported higher EPG in diarrheic samples compared to normal pelleted faeces in lambs but they observed reverse trend in adult sheep 
Faecal Consistency: A Measure of Gastrointestinal Parasitic Infections in Goats

Table 1: Effect of variations on faecal oocysts and egg counts in goat with gastrointestinal parasitic infections.

\begin{tabular}{|c|c|c|c|c|c|}
\hline Traits/effects & $\begin{array}{l}\text { Total } \\
\text { Obs. }\end{array}$ & $\begin{array}{l}\text { Log transformed } \\
\text { Coccidian OPG }\end{array}$ & $\begin{array}{c}\text { Geometric mean } \\
\text { of OPG }\end{array}$ & $\begin{array}{l}\text { Log transformed } \\
\text { Strongyles EPG }\end{array}$ & $\begin{array}{c}\text { Geometric mean } \\
\text { of EPG }\end{array}$ \\
\hline Overall mean & 1451 & $4.767 \pm 0.010$ & 1466 & $4.621 \pm 0.001$ & 318 \\
\hline Sample types & & ** & & ** & \\
\hline Normal & 867 & $4.707 \pm 0.013^{a}$ & 2034 & $4.614 \pm 0.002^{\mathrm{a}}$ & 176 \\
\hline Lumpy & 358 & $4.763 \pm 0.017^{b}$ & 3418 & $4.622 \pm 0.002^{b}$ & 338 \\
\hline Diarrhoic & 226 & $4.831 \pm 0.021^{c}$ & 5066 & $4.625 \pm 0.003^{b}$ & 400 \\
\hline Age & & ** & & ** & \\
\hline$<3 \mathrm{M}$ & 182 & $4.866 \pm 0.023^{a}$ & 5960 & $4.611 \pm 0.003^{a}$ & 116 \\
\hline$>3-6 \mathrm{M}$ & 353 & $4.780 \pm 0.017^{b}$ & 3820 & $4.612 \pm 0.002^{\mathrm{a}}$ & 136 \\
\hline$>6-12 \mathrm{M}$ & 307 & $4.739 \pm 0.021^{\mathrm{b}}$ & 2864 & $4.628 \pm 0.003^{b}$ & 460 \\
\hline Adult(>1Y) & 609 & $4.683 \pm 0.018^{c}$ & 1618 & $4.631 \pm 0.002^{b}$ & 524 \\
\hline Sample type $X$ Age & & ** & & NS & \\
\hline Normal $X<3 M$ & 56 & $4.704 \pm 0.041^{\mathrm{cd}}$ & 2076 & $4.607 \pm 0.005$ & 36 \\
\hline Normal $\mathrm{X}>3-6 \mathrm{M}$ & 177 & $4.752 \pm 0.023^{\mathrm{bc}}$ & 3162 & $4.611 \pm 0.003$ & 116 \\
\hline Normal $X>6-12 M$ & 197 & $4.688 \pm 0.022^{d}$ & 1726 & $4.621 \pm 0.003$ & 318 \\
\hline Normal X Adult & 437 & $4.682 \pm 0.015^{\mathrm{d}}$ & 1596 & $4.618 \pm 0.002$ & 258 \\
\hline Lumpy $\mathrm{X}<3 \mathrm{M}$ & 56 & $4.842 \pm 0.041^{\mathrm{b}}$ & 5344 & $4.611 \pm 0.005$ & 116 \\
\hline Lumpy $\mathrm{X}>3-6 \mathrm{M}$ & 106 & $4.791 \pm 0.030^{\mathrm{bc}}$ & 4084 & $4.614 \pm 0.004$ & 176 \\
\hline Lumpy $X>6-12 M$ & 67 & $4.726 \pm 0.037^{c d}$ & 2568 & $4.627 \pm 0.005$ & 442 \\
\hline Lumpy X Adult & 129 & $4.692 \pm 0.027^{d}$ & 1814 & $4.637 \pm 0.004$ & 646 \\
\hline Diarrheic $X<3 M$ & 70 & $5.051 \pm 0.036^{a}$ & 11234 & $4.617 \pm 0.005$ & 238 \\
\hline Diarrheic $X>3-6 M$ & 70 & $4.796 \pm 0.036^{\mathrm{bc}}$ & 4204 & $4.612 \pm 0.005$ & 136 \\
\hline Diarrheic $X>6-12 M$ & 43 & $4.801 \pm 0.046^{b}$ & 4326 & $4.636 \pm 0.006$ & 626 \\
\hline Diarrheic X Adult & 43 & $4.676 \pm 0.046^{d}$ & 1466 & $4.637 \pm 0.006$ & 646 \\
\hline
\end{tabular}

Note: Least squares means carrying different alphabet differed significantly $(P<0.01)$. ${ }^{* *}$ means Significant $(P<0.01)$, NS Non-significant.

and attributed scouring in adults is due to hypersensitivity to ingested nematodes larvae instead of existing worm load. Seyoum et al. (2018) observed an inverse relationship between faecal consistency and GI nematode FEC and they attributed it to more water in faeces.

The higher EPG and OPG in diarrheic sample types are suggestive that both coccidian and strongyle infections were associated with diarrhoea. The results in the study were important and carried significance because the study was based on faecal samples collected and examined throughout the year whiles all other factors being common. Further, the result suggested that the FCS could be an important predictor of the intensity of gastrointestinal parasitism (Abebe, 2010, Bentounsi et al. 2012).

Faecal consistency with increased moisture dilutes the per gram faecal eggs/oocysts counts and the observed EPG/ OPG values need correction (Le Jambre et al. 2007; Gordon, 1967). Gordon (1967) classified faecal samples, on appearance basis, into a series of five consistency grades and suggested multiplier factors for each to correct the dilution effect of diarrhoea. Le Jambre et al. (2007) used a 6 -grade scale to classify the faecal samples and studied the relationship between FCS and simulated worm egg counts (WEC) and dry matter. They testified the adjustment factors of Gordon on a dry matter basis by faecal consistency score of 4.0 and described that WEC and susceptibility of sheep may be underestimated with increased FCS. In contrast, Turner et al. (2010) reported a positive relationship between faecal water content and faecal egg counts and described that faecal water content may have a limited potential to confound estimates of parasite intensity from per gram faecal egg counts in free-range systems.

The present study differed from Gordon (1967) and Le Jambre et al. (2007) in the number of faecal consistency grades/scores being 3 as compared to 5 and 6 respectively in their studies. The 3 grades of faecal consistency scores here were similar to various other studies (Ghimire and Bhattarai 2019; Turner et al. 2010; Seyoum et al. 2018; Bentounsi et al. 2012; Gibbons et al. 2016). The 3 grade scores, in this study, were more practical for ease of observations recording because a wide range of faecal consistency grades/scores may likely create confusion increasing the chances of overlapping in its use. Whereas, Jacobson et al. (2009) used only two faecal consistency grades i.e. normal and scouring.

Theory of dilution of Gordon (1967) and Le Jambre et al. (2007) might have significance when the FEC is considered as a selection tool for worm resistance animals because confounding can affect estimated breeding values. However, in animals suffering from diarrhoea and also showing significantly more EPG/OPG, the FCS provide sufficient ground to go for selective drenching treatment 


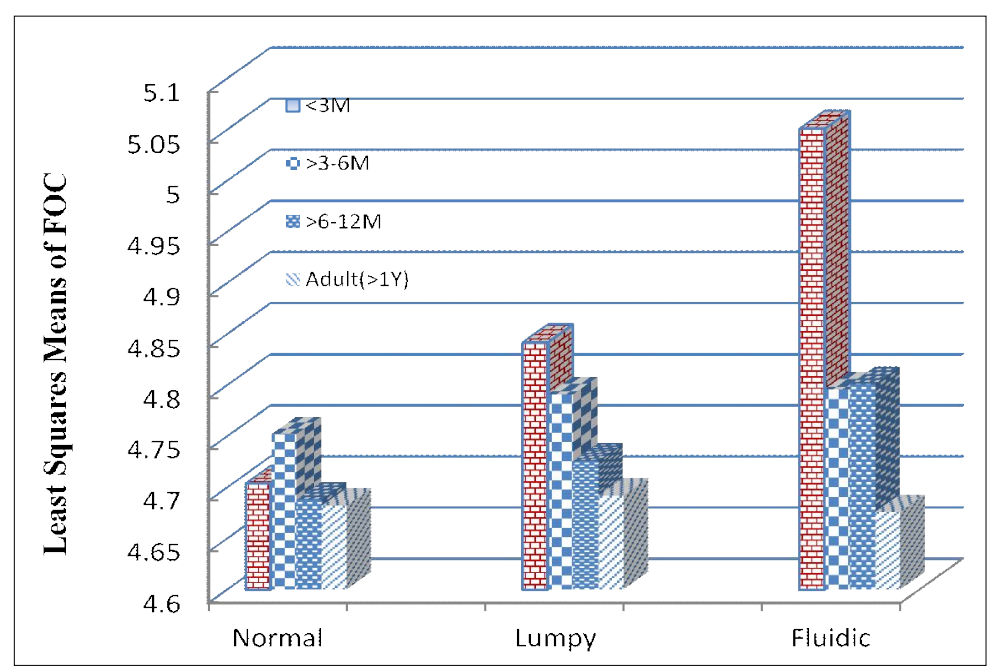

Fig 2: Least squares means representing interaction between faecal sample types and age for FOC.

(Broughan and Wall (2007). Further, in the light of anorexia, with reduced feed intake, being the consistent finding in all types of intestinal parasitic infections (Smith and Sherman, 2009 ) it is to be studied if faecal consistency in diarrhoea is a result of dilution or simply proportional change of dry matter and water content. In later case, however, FEC/FOC would hardly ever change.

Results of the study suggested that severity of coccidian infection (higher OPG) was more in young ones (especially in $<3$ months age) than the adults (Sharma et al. 2017; Kaur et al. 2019) and supported the descending trend of per gram FOC with ascending age as described by Smith and Sherman (2009). In contrast to Eimeria infection, mean EPG of strongyle worms was significantly higher in goats above 6 months of age (Sharma et al. 2009; Sharma et al. 2013; Dappawar et al. 2018). Analysis of data showed that the problem of strongyle worms was of more importance in goats after 6 month age.

As age plays an important role in determining the rate of prevalence and severity of coccidian infection as well as strongyle infection in animals, the effect of interaction between faecal sample types and animal age was considered to analyze. The effect of interaction of sample types $X$ animal age about FEC (strongyle worms) in the present study was not significant. This can be explained because Haemonchus contortus being the major GI nematode infection in present study where diarrhoea was not a consistent symptom. In contrast, Bentounsi et al. (2012) described diarrhoea score as the most effective indicator for identifying sheep, which need treatment. The variation here can also be attributed to the diversified age of animals in the study.

The effect of the interaction of sample types $X$ animal age about coccidian FOC was, however, highly significant. These results were of importance as interaction means of per gram FOC in all faecal sample types for young age (less than 1 year) were significantly higher than old age (above 12M) (Table-1)(Fig 2). Further, this was more important in diarrheic samples to suggest that faecal consistency might be a measure of coccidian infection in goats, especially in young kids and also a guiding tool for selective treatment.

\section{CONCLUSION}

The study concluded that consistency of faecal matter, being a result of various factors involving feed, fodder, seasons and state of gut physiology of digestion, may be affected the due presence of gastrointestinal parasitic infections like strongyle worms and coccidia, the known causes to result diarrhoea and enteritis. The study conducted in the organized flock with similar management conditions throughout the year carries importance for farmers and farm owner for deciding anti-coccidian treatment in young goats. However, similar studies, with more number of faecal samples analyzed, would help to buttress the hypothesis in a better way.

\section{ACKNOWLEDGEMENT}

Authors do sincerely acknowledge the technical and financial helps from Director, CIRG, Makhdoom, Farah, Mathura, (UP) India to complete the study.

\section{Conflict of authors interest}

No conflict of interest among authors exists and all the authors have seen the manuscript and consented for submission.

\section{Declaration/ statement of animal rights}

It is declared that there is no ethical issue involved in the study as no live animal has been part of it.

\section{REFERENCES}

Abebe, R., Gebreyohannes, M., Mekuria, S., Abunna, F. and Regassa, A. (2010). Gastrointestinal nematode infections in small ruminants under the traditional husbandry system during the dry season in southern Ethiopia. Tropical Animal and Health Production. 42: 1111-1117. 
Faecal Consistency: A Measure of Gastrointestinal Parasitic Infections in Goats

APHA, AWWA and WEF. (2005). Standard methods for the examination of water and wastewater. $21^{\text {st }}$ ed. Washington, DC: American Public Health Association, American Water Works Association and Water Environment Federation; p. 7-15.

Bayew, K. (2019). Prevalence of coccidiosis in calves. International Journal of Agriculture and Agribusiness. 292: 249-256.

Bentounsi, B., Meradi, S. and Cabaret, J. (2012). Towards finding effective indicators (diarrhea and anaemia scores and weight gains) for the implementation of targeted selective treatment against the gastro-intestinal nematodes in lambs in a steppic environment. Veterinary Parasitology. 187(1-2): 275-279.

Broughan, J.M. and Wall, R. (2007). Faecal soiling and gastrointestinal helminth infection in lambs. International Journal of Parasitology. 37: 1255-1268.

Burke, J.M., Kaplan, R.M., Miller, T.H., Terrill, T.H., Getz, W.R., Mobini, S., Valencia, E., William, M.J., Williamson, L.H. and Vatta, A.F. (2007). Accuracy of FAMACHA system for on-farm use by sheep and goat producers in southeastern United States. Veterinary Parasitology. 147: 89-95.

Cintra, M.C.R., Ollhoff, R.D. and Sotomaior, C.S. (2018). Sensitivity and specificity of the FAMACHA System in growing lambs. Veterinary Parasitology. 251(2): 106-111.

Dappawar, M.K., Khillare, B.S., Narladkar, B.W., Bhangale, G.N. (2018). Prevalence of gastrointestinal parasites in small ruminants in Udgir area of Marathwada. Journal of Entomology Zoology Studies. 6(4): 672-676.

Gaddam, M.J. (2005). Epidemiology of coccidiosis in calves and control of coccidiosis using Toltazuril at the time of weaning. A Thesis of Master of Philosophy in Veterinary Parasitology submitted to Massey University, Palmerstone North, New Zealand.

Ghimire, T.R. and Bhattarai, N. (2019). A survey of gastrointestinal parasites of goats in a goat market in Kathmandu, Nepal. Journal of Parasitic Diseases. 43(4): 686-695.

Gibbons, P., Love, D., Craig, T. and Budke, C. (2016). Efficacy of treatment of elevated coccidial oocyst counts in gats using amprolium versus ponazuril. Veterinary Parasitology. 218: $1-4$

Gonzalez-Garduno, R., Lopez-Arellano, M.E., Torres-Hernandez, G., Oliva-Hernandez, J. and Hinojosa-Cuellar, J.A. (2018). Assessment of acquired resistance in previously infected lambs with gastrointestinal nematodes in a tropical climate Indian Journal of Animal Research. 53(9): 12231228.

Gordon, H.M. (1967). The diagnosis of helminthosis in sheep. Vet. Med. Rev., Leverkusen, (2/3), 140-168.

Harvey, W.R. (1990). User's guide for LSMLMW PC-2 Version mixed model least-squares maximum likelihood computer program. Minneograph Columbus, Ohio, USA.

Hassum, I.C. (2014). FAMACHA method as tool for selective control of nematode parasites in sheep. Revista Brasileiria de Medicina Veterinaria. 36(3): 251-254.

Jacobson, C.L., Bell, K. and Besier, R.B. (2009). Nematode parasites and faecal soiling of sheep in lairage: Evidence of widespread potential production losses for the sheep industry. Animal Production Science. 49: 326-332.
Kaplan, R. M., Burke, J.M., Terrill, T.H., Miller, J. E., Getz, W.R., Mobini, S., Valencia, E., Williams, M.J., Williamson, L.H., Larsen, M. and Vatta. A.F. (2004). Validation of the FAMACHA eye color chart for detecting clinical anemia in sheep and goats on farms in the southern United States. Veterinary Parasitology. 123(1-2): 105-120.

Kaur, S., Singla, L.D., Sandhu, B.S., Bal, M.S. and Kaur, P. (2019). Coccidiosis in goats: Pathological observations on intestinal developmental stages and anticoccidial efficacy of amprolium. Indian Journal of Animal Research. 53: 245-249.

Kramer, C.Y. (1957). Extension of multiple range tests to group correlated adjusted means. Biometrics. 13(1): 13-18.

Le Jambre, L.F., Dominik, S., Eady, S.J., Henshell, J.M., Colditz, G.A. (2007). Adjusting worm egg counts for faecal moisture in sheep. Veterinary Parasitology. 145(1-2): 108-115.

McEwan, J.C., Mason, P., Baker, R.L., Clarke, J.N., Hickey, S.M., Turner, K. (1992). Effect of selection for productive traits on internal parasite resistance in sheep. Proceeding of New Zealand Society of Animal Production. 52: 53-56.

MOFF. (1977). Manual of Veterinary Parasitology Laboratory Technique. Technical Bulletin No.18.pp 160, Her Majesty's Stationery Office, Ministry of Agriculture, Fisheries and Food, London.

Reynecke, D., Van Wyk, J.A., Gummow, B., Dorny, P. and Boomker, J. (2009). Validation of the FAMACHA@ eye colour chart using sensitivity/ specificity analysis on two South African sheep farms. Veterinary Parasitology. 177(3-4): 203-11. DOI: 10.1016/j.vetpar. 2009.08.023.

Saratsis, A., Joachim, A., Alexandros, S. and Sotiraki, S. (2011). Lamb coccidiosis dynamics in different dairy production systems. Veterinary Parasitology. 181: 131-138.

Schlink, A.C., Nguyen, M.L. and Viljoen, G.J. (2010). Water requirements for livestock production: A global perspective. Revue Scientifique et Technique Office International des Epizooties. 29(3): 603-619.

Seyoum, Z., Getnet, K., Chanie, M., Derso, S. and Fentahun, S. (2018). Morbidity Parameters Associated with Gastrointestinal tract nematodes in sheep in Dabat District, Northwest Ethiopia. BioMed Research International. Article ID 9247439, doi.org/10.1155/2018/9247439.

Sharma, D.K. and Mandal, A. (2013). Factors affecting gastrointestinal parasite infections in goats in semi-arid rural ecosystems in India. Veterinary Science Development. DOI: 10.4081/ vsd.2013.e5.

Sharma, D.K., Agrawal, N., Mandal, A., Nigam, P. and Bhushan, S. (2009). Coccidia and gastrointestinal nematode infections in semi-intensive managed Jakhrana goats of semi-arid region of India. Tropical Subtropical Agroecosystems. 11(1): 135-139.

Sharma, D.K., Paul, S., Rout, P.K., Mandal, A., Bhusan, S., Sharma, N. and Kushwah, Y.K. (2017). Caprine coccidiosis in semi-arid India: Dynamics and factors affecting faecal oocysts count. Journal of Advanced Veterinary and Animal Research. 4(1): 52-57.

Smith M.C. and Sherman M.D. (2009). Goat Medicine. $2^{\text {nd }}$ Edition, Published by Wiley-Blackwell, 2121 State Avenue, Ames, lowa 50014-8300 USA. 
Torres-Acosta, J.F.J., Perez-Cruz, M., Canul-Ku, H.L., SotoBarrientos, N., Camara-Sarmiento, R., Aguilar-Caballero, A.J., Lozano-Argaes, I., Le-Begot, C. and Hoste, H. (2014). Building a combined targeted selective treatment scheme against gastrointestinal nematodes in tropical goats. Small Ruminant Research. 121(1): 27-35.

Turner, W.C., Cizauskas, C.A. and Getz, W.M. (2010). Variation in faecal water content may confound estimates of gastrointestinal parasite intensity in wild African herbivores. Journal of Helminthology. 84(1):99-105 doi: 10.1017/ S0022149X09990320.
Waghorn, G.C., Gregory, M.G., Todd, S.E. and Wesselink, R. (1999). Dag in sheep: A look at faeces and reasons for dag formation. Proceeding of the New Zealand Grassland Association. 61: 43-49.

Wesselink, R., Waghorn, G.C. and McNabb, W.C. (1995). Causes of dagginess in sheep: A survey of the literature undertaken for Wools of New Zealand. Palmerston North, AgResearch Grasslands. 